\title{
AUTHOR INDEX TO VOLUME 29, 2004
}

\section{【A】}

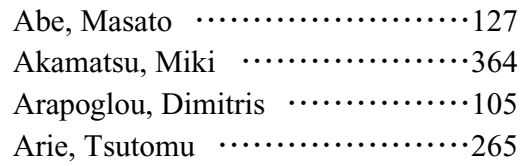

【B】

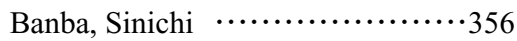

\section{【C】}

Casida, John E. ..................8

\section{【F】}

Fujihara, Shinsuke $\cdots \cdots \cdots \cdots \cdot \ldots \cdot \ldots 3$

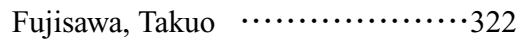

Fukuchi, Atsushi $\quad$ …..............209

Fusaka, Takafumi $\ldots . . . \ldots \ldots \ldots . \cdots 339$

\section{【H】}

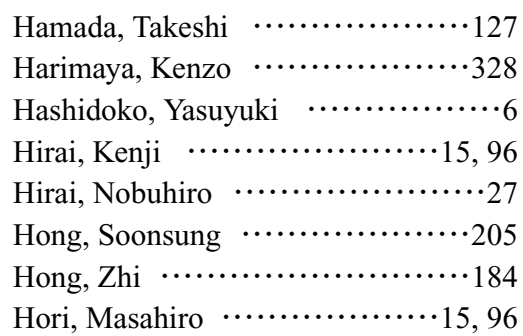

【I】

Ichimaru, Naoya $\cdots \cdots \cdots \cdots \cdot \ldots \cdot \cdots \cdot \cdots \cdot 127$

Ichise-Shibuya, Keiko ….........322

Ikeda, Mitsumasa

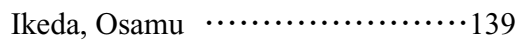

Imamura, Kei-ichi $\cdots . . . \ldots \ldots \ldots . . . \cdots 328$

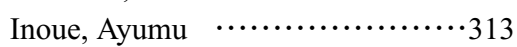

Isayama, Shinji $\quad \cdots \ldots \ldots \ldots \ldots \ldots . \cdots \cdot 372$

Ishihara, Atsushi $\cdots \cdots \cdots \cdots \cdot \ldots \cdot 217,227$

Ishikawa, Ryo ..................209

Islam, Md. Tofazzal $\cdots \cdots \cdots \cdots \cdots \cdots \cdot \ldots$

Israilides, Cleanthes $\cdots \cdots \cdots \cdots \cdot \cdots \cdot \cdots \cdot 105$

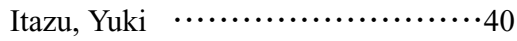

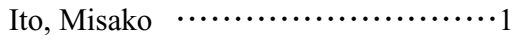

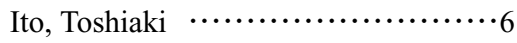

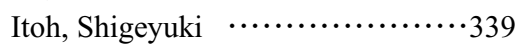

Iyozumi, Hiroyuki $\cdots \cdots \cdot \ldots \cdot \cdots \cdot \cdots \cdot 256$

【J】

Jikihara, Tetsuo 139

\section{【K】}

Kagabu, Shinzo $\cdots \cdots \cdots \cdot \cdots \cdot \cdot 40,43,376$

Kajiwara, Takeshi $\quad \cdots . . . . . . . \cdots \cdots \cdot . \cdot 339$

Kamada, Takashi $\quad \cdots \cdot \ldots \ldots \ldots \ldots \cdot \cdots \cdot \cdots \cdot 272$

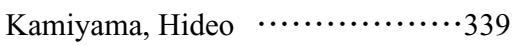

Karlis, Panagiotis $\cdots \cdot \ldots \ldots . . \cdots \cdots \cdot 105$

Kasai, Shinji $\quad \cdots \cdots \cdots \cdots \cdot \ldots \cdot \cdots \cdot 220,234$

Katagi, Toshiyuki $\cdots \cdots \cdot \ldots \cdot \ldots 322,332$

Kataoka, Yuki ….................205

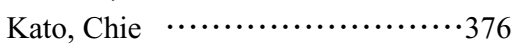

Kawahara, Nobuyuki …..........348

Kawai, Shinya $\quad \cdots \cdots \cdots \cdot \ldots \ldots \cdot \cdots \cdot \cdots \cdot 205$

Kenmochi, Atsushi $\quad \cdots \cdots \cdots \cdot \ldots \cdot \cdots \cdot 127$

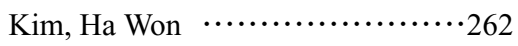

Kimura, Yukio $\cdots \cdots \cdots \cdot \ldots \ldots . \cdots \cdot 195$

Kinoshita, Katsutoshi $\cdots \cdots \cdot \cdots 348,356$

Kinoshita, Mitsuaki …...........395

Kiriyama, Kazuhisa $\cdots \cdots \ldots \ldots \ldots . \cdots 43$

Kodaka, Kenji $\cdots \cdots \cdots \cdots \cdot \ldots \cdot \cdots 348,356$

Kodaka, Rika ....................332

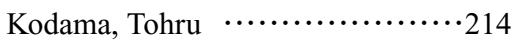

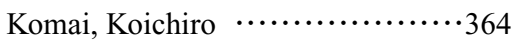

Komyoji, Terumasa $\cdots \cdots \cdots \cdots \cdots \cdot \ldots \cdot 136$

Kono, Yoshiaki …...............60

Kuriyama, Tadahiko $\cdots \cdot \cdots \cdot \cdots \cdot \cdots \cdot \cdots 328$

Kuwahara, Yasumasa $\cdots \cdots \cdots \cdot \ldots \cdot \cdots \cdot 27$

Kuwano, Eiichi $\cdots \cdots \cdots \cdots \cdot \cdots \cdot 121,205$

\section{【L】}

Leichter, Cheryl A. $\quad \cdots \cdots \cdots \cdots \cdots \cdot \cdots \cdot 124$

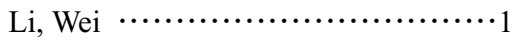

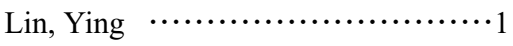

\section{【M】}

Mabuchi, Tsutomu ................195

Makino, Takahiro …..............256

Manabe, Akio ...................245

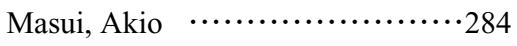

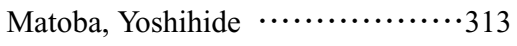

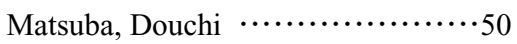

Matsuda, Kazuhiko $\cdots \cdot \cdots \cdot \ldots \cdot \cdots \cdot \cdots \cdot 364$

Matsukawa, Tomoko $\cdot \cdots \cdots \cdots \cdots \cdot \ldots \cdot \cdots \cdot 15$

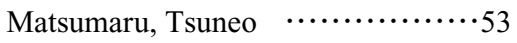

Matsumoto, Hiroshi $\quad \cdots \cdot \ldots . \cdots \cdots \cdot 117$

Matsumura, Fumio …........299, 402

Matsuo, Norifusa $\cdots . . . . . \cdots \cdots \cdot \ldots 136$

Matsuoka, Makoto $\quad \cdots \cdot \ldots . \cdots \cdots \cdot \ldots \cdot \cdots 184$

Matsuura, Kazuho ….............209

Minakuchi, Chieka ..............189

Mitani, Shigeru $\cdots \cdots \cdot \ldots \ldots \ldots \ldots \cdot 136$
Miura, Ichirou ….................369

Miura, Yuzo ….................195

Miyagawa, Hisashi $\cdots \cdots \cdot \ldots \cdot \cdots \cdot 110,189$

Miyashita, Masahiro $\quad \cdots \cdot \ldots \cdot \cdots \cdot \cdots \cdot 110$

Miyoshi, Hideto

Mizutani, Hiroyuki …..............87

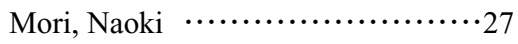

Morita, Atsushi $\quad \cdots \cdot \ldots . . . \cdots \cdots \cdot \ldots \cdot \ldots 27$

Munakata, Toshio $\quad \cdots \cdot \ldots . . . \cdots \cdots \cdot \ldots 139$

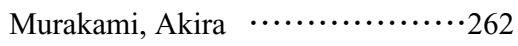

Murata, Shinji …................. 195

Murata, Yoshiyuki …............304

\section{【N】}

Nagami, Takamichi $\cdots \cdot \cdots \cdot \cdots \cdot \cdots \cdot \cdots \cdot 205$

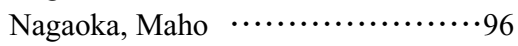

Nagayama, Kozo .................369

Nakagawa, Yoshiaki $\quad \cdots \cdots \cdots \cdot \cdots 110,189$

Nakajima, Toshio $\cdot \cdots \cdot \ldots . . \cdots \cdots \cdot \ldots \cdot 136$

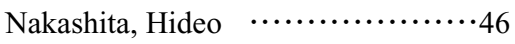

Nakayama, Kayo ................200

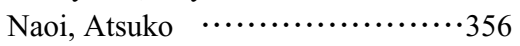

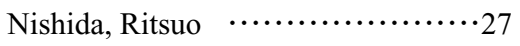

Nishimura, Keiichiro $\cdots \cdots \cdot 40,43,376$

Nishioka, Takaaki $\quad \cdots \cdots \cdots \cdot \ldots \cdot \cdots \cdot 127$

Nishiwaki, Hisashi $\cdots \cdots \cdot 110,222,240$

Noda, Hiroaki $\quad$...................... 163

Noma, Masana $\quad \cdots \cdot \ldots \ldots \ldots \ldots \ldots \cdot 200$

\section{【o】}

Ohigashi, Hajime $\quad \cdots \cdots \cdot \ldots . \cdots \cdots \cdot \ldots 262$

Ohno, Ryuta $\quad \cdots \cdots \cdots \cdots \cdots \cdots \cdot \cdots \cdot \cdots \cdot 15,96$

Ohshima, Takeshi $\quad \cdots . . . . . . . . \cdots \cdots \cdot 136$

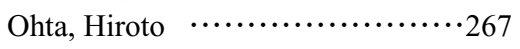

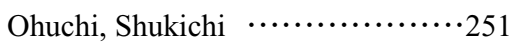

Okano, Ruiko ….................50

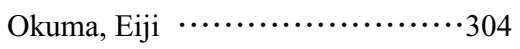

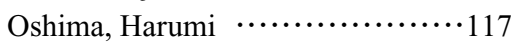

Otani, Toshikazu …................33

Oyama, Kazuhiko ................3 328

Ozoe, Yoshihisa $\cdots \cdots \cdots \cdot \cdots \cdot 134,267,328$

\section{【Q】}

Quistad, Gary B. $\cdot 81$

\section{【R】}

Rinkevich, Frank D. 124

\section{【S】}

Saito, Isao $\cdots \cdots \cdots \cdots \cdots \cdot \cdots \cdot 117,394,398$

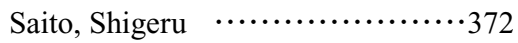




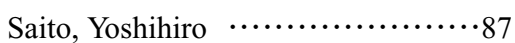

Sakai, Michihiko $\quad \cdots . . . . . . . . . . . . .288$

Sakamoto, Noriyasu

Sakurai, Hiroshi $\quad \cdots \cdots \cdots \cdots \cdots \cdot \cdots \cdot 15,96$

Sánchez-Bayo, Francisco $\cdots \cdots \cdots \cdot \cdots \cdot 130$

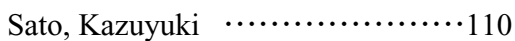

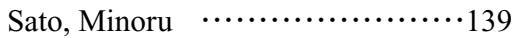

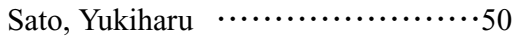

Sattelle, David B. $\cdots \cdots \cdots \cdots \cdots \cdot \cdots \cdot \cdots \cdot \cdots$

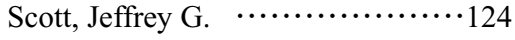

Shibuya, Naoto $\cdots \cdots \cdots \cdot \ldots \cdot \cdots \cdots \cdot \cdots \cdot 276$

Shimizu, Ryo

Shimomura, Masaru …...........364

Shiotsuki, Takahiro $\cdots \ldots \ldots \ldots \ldots \ldots . \cdots 121$

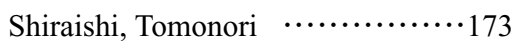

Soh, Kazuhiro ...................396

Soya, Yoshihiro $\cdots \cdots \ldots \ldots \ldots \ldots \ldots . \cdots 189$

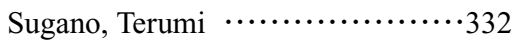

Sugawara, Fumio $\quad \cdots \cdots \cdots \cdots \cdots \cdots \cdot \ldots \cdot \cdots$

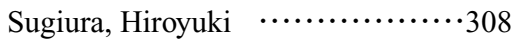

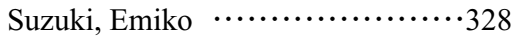

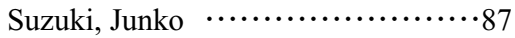

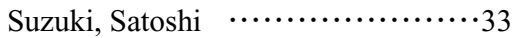

Suzuki-Nishimito, Mayumi $\cdots \cdots \cdot \cdot 209$

\section{【T】}

Tachibana, Yuji $\ldots \ldots \ldots \ldots \ldots \ldots \ldots . . . \cdots 328$

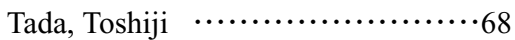

Tahara, Satoshi $\cdots \ldots \ldots \ldots \ldots \ldots \ldots \cdot 6$

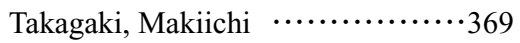

Takahashi, Nobutoshi $\quad \cdots \cdots \cdots \cdot \cdots \cdot \cdots 328$

Takazaki, Hirotoshi $\cdots \cdots \cdots \cdot \ldots \cdot \cdots \cdot \cdots 50$

Takimoto, Yoshiyuki $\cdots \cdot 313,322,332$

Tamogami, Shigeru …............200

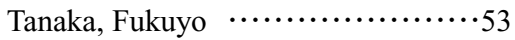

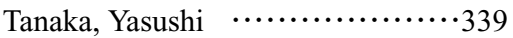

Tokumaru, Susumu $\cdots \cdots \cdots \cdots \cdots \cdots \cdots \cdots$

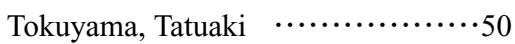

Tomizawa, Motohiro …....................

Torra-Reventós, Mercè …..........214

Tsuchiya, Hiroshi

Tsuzuki, Manabu $\cdots \cdot \ldots \cdot \ldots \cdot \cdots \cdot \cdots \cdot 332$

\section{【U】}

Ueda, Takuya $\cdots \cdots \cdots \cdots \cdot \cdots \cdot \cdots \cdot \cdots \cdot 15,96$

Ueguchi-Tanaka, Miyako $\cdots \cdots \cdot \cdots \cdot 184$

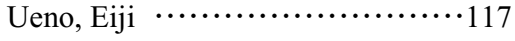

Ujita, Minoru

Umeda, Kimitoshi ................372
Uozaki, Tatsuhiko 397

\section{【V】}

Vlyssides, Apostolos $\cdots \cdots \cdots \cdots \cdot \cdots \cdot 105$

【W】

Wakabayashi, Ko $\quad \cdots \cdots \cdots \cdot \cdots \cdot \cdots \cdot \cdots \cdot \cdots$

Wakita, Takeo $\cdots \cdots \cdots \cdot \ldots \cdot \cdots \cdot \cdots 348,356$

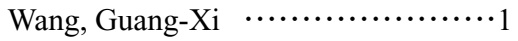

Watanabe, Atsuko $\cdots \cdots \cdots \cdots \cdots \cdot 15,96$

Williams, Marshall V. ….........262

\section{【Y】}

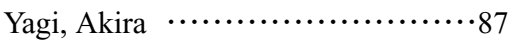

Yaguchi, Takashi $\quad$ …...............328

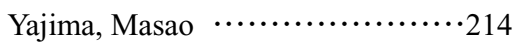

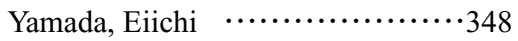

Yamada, Naotaka …...............205

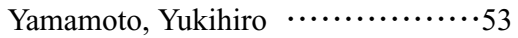

Yamanaka, Shigeru $\cdots \cdots \cdots \cdots \cdot \cdots \cdot \cdots \cdot 214$

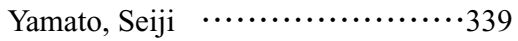

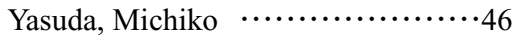

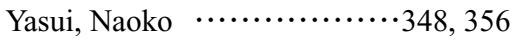

Yokota, Toshiyasu …..............395

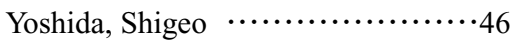

